1 Humphris et al. ${ }^{2}$ have just published a paper looking at health and wellbeing as an outcome of uncertainties of the pandemic in dental team members. 'Of 329 respondents the researchers report that $27 \%$ appear to have reported significant depressive symptomology and 55\% appear to suggest that they have suffered emotional exhaustion. ${ }^{2}$ Interestingly, the paper suggests that primary care staff reported higher incidences. The recommendations clearly suggest that there could be benefit in 'resourcing staff support and interventions to help team members in [challenging times].'

Not only does the Humphris et al. ${ }^{2}$ paper speak to the whole team, it touches on the narrative of resourcing staff support and interventions, which takes this full circle and right back to the principles of the extensive work recently undertaken by the Dental Professional Alliance and stakeholders, who have designed a framework introducing the need for a mental health wellness lead in every dental setting with an underlying ethos that early intervention and safe signposting is paramount.

The initial call to action from the initiative is that each dental workplace should have an identified individual who acts as a 'mental health wellness lead' and who through a recognised training programme is confident, competent and committed to improving the perception of mental health wellness in the workplace. This forms part of a six-part process:
- Identify mental health wellness lead

- All members of dental team to undergo stress awareness training

- Leads to undertake MHFA training

- Design workplace action plan

- Join local peer support networks

- Complete annual training and maintain skills.

Roz McMullan said, 'No one should feel alone or unable to talk to someone at work and for this very reason, this "call to action" asks decision makers and line managers to adopt this cultural change to mental health wellness in the dental workplace, and commit to the recognised training pathway'.

For more information about the initiative and how you can get involved, visit: https://mhwd.org/.

\section{References}

1. Office for National Statistics. Coronavirus and depression in adults, Great Britain: January to March 2021. Analysis of the proportion of the British adult population experiencing some form of depression in early 2021 by age, sex and other characteristics. Includes comparisons with 2020 and pre-pandemic estimates. 5 May 2021. Available at: https://www.ons.gov.uk/peoplepopulationandcommunity/ wellbeing/articles/coronavirusanddepressioninadultsgreatbritain/ januarytomarch2021 (accessed May 2021).

2. Humphris G, Knights J, Beaton L et al. Exploring the effect of the COVID-19 pandemic on the dental team: preparedness, psychological impacts and emotional reactions. Front Oral Health 2021 https://doi.org/10.3389/froh.2021.669752.

\title{
GDC publishes statement on direct-to-consumer orthodontics
}

The General Dental Council (GDC) has published an updated statement ${ }^{1}$ in relation to direct-to-consumer orthodontics which highlights issues dental professionals - particularly those working in the remote provision of orthodontics - need to consider.

The statement is based on three main requirements:

1. In line with current authoritative clinical guidance, and orthodontic training, clinical judgements about the suitability of a proposed course of orthodontic treatment must be based on a full assessment of the patient's oral health. At present, there is no effective substitute for a physical, clinical examination as the foundation for that assessment. Should a dentist rely upon information from another source to inform their own clinical judgement, the responsibility for that judgement rests wholly with the prescribing dentist

2. Direct interaction between patient and practitioner - whether in person or remotely - is essential for providing patients with the opportunity to ask questions, provide valid and informed consent, and be satisfied that the course of treatment proposed is likely to meet their needs and expectations

3. Patients must know the full name of the dental professional responsible for their treatment and be able to make direct contact with that person if they need to.

GDC Executive Director, Strategy, Stefan Czerniawski, said: 'Traditionally, healthcare is delivered in person - patient and clinician sitting in the same room discussing treatment options and agreeing on a way forward. New technology is challenging that norm and we support innovation wherever it safely brings benefits to the patient. But safety is absolutely key and new ways of doing things must not compromise well-established safety measures which are designed to protect patients.

'Our statement highlights a number of issues that dental professionals working in remote models of treatment will need to consider. One of the main things to remember though, as is the case in any other setting, it remains the treating dentist who is responsible for ensuring they have met all of the Standards for the dental team.

In addition to the statement, the GDC has also published information to support dental professionals in exercising professional judgement. ${ }^{2}$

Should professionals have any questions or concerns in relation to their practice, they should seek further advice from their indemnifier or professional association. The GDC says it will continue to monitor developments in this area.

\section{References}

1. General Dental Council. GDC Statement on 'direct-to-consumer' orthodontic treatment. Available at: https://www.gdc-uk.org/information-standardsguidance/standards-and-guidance/gdc-guidance-for-dental-professionals/ direct-to-consumer-orthodontics/gdc-statement-on-direct-to-consumerorthodontic-treatment (accessed 17 May 2021)

2. General Dental Council. Direct-to-consumer orthodontics: information to support professional judgement. Available at: https://www.gdc-uk.org/informationstandards-guidance/standards-and-guidance/gdc-guidance-for-dentalprofessionals/direct-to-consumer-orthodontics (accessed 17 May 2021). 